Research Article

\title{
Combined Treatment of Cinobufotalin and Gefitinib Exhibits Potent Efficacy against Lung Cancer
}

\author{
Youxi Han $\mathbb{D}^{1},{ }^{2}$ Ronghui Ma $\mathbb{D}^{2},{ }^{2}$ Guolei Cao $\mathbb{D}^{2},{ }^{2}$ Hao Liu $\left(\mathbb{D},{ }^{3}\right.$ Lili He $\mathbb{D}^{2},{ }^{2}$ Le Tang $\left(\mathbb{D},{ }^{2}\right.$ \\ Hong Li $\mathbb{D}^{2}{ }^{2}$ and Qin Luo $\mathbb{D}^{2}$ \\ ${ }^{1}$ Department of Breast Radiotherapy, The Affiliated Tumor Hospital of Xinjiang Medical University, Urumqi 830000, China \\ ${ }^{2}$ Department of Respiratory Neurology, The Affiliated Tumor Hospital of Xinjiang Medical University, Urumqi 830000, China \\ ${ }^{3}$ Department of Infection, The Affiliated Tumor Hospital of Xinjiang Medical University, Urumqi 830000, China
}

Correspondence should be addressed to Qin Luo; maronghui0204@163.com

Received 7 November 2020; Revised 8 March 2021; Accepted 10 March 2021; Published 20 March 2021

Academic Editor: Mateus R. Beguelini

Copyright (c) 2021 Youxi Han et al. This is an open access article distributed under the Creative Commons Attribution License, which permits unrestricted use, distribution, and reproduction in any medium, provided the original work is properly cited.

This study aimed to evaluate the efficacy of cinobufotalin combined with gefitinib in the treatment of lung cancer. A549 cells were treated with gefitinib, cinobufotalin, or cinobufotalin plus gefitinib. MTT assay, annexin-V/PI staining and flow cytometry, TUNEL staining, DCFH-DA staining, Western blot, and real-time RT-PCR were performed to investigate the synergistic inhibitory effect of cinobufotalin combined with gefitinib on the growth of A549 cells. Results showed that cinobufotalin synergized with gefitinib displayed inhibited cell viability and enhanced apoptosis in the combination group. Cinobufotalin combined with gefitinib induced a significant enhancement in reactive oxygen species (ROS) production accompanied by cell cycle arrest in the $\mathrm{S}$ phase arrest, characterized by upregulation of p21 and downregulation of cyclin A, cyclin E, and CDK2. Besides, cinobufotalin plus gefitinib downregulated the levels of HGF and c-Met. In summary, cinobufotalin combined with gefitinib impedes viability and facilitates apoptosis of A549 cells, indicating that the combined therapy might be a new promising treatment for lung cancer patients who are resistant to gefitinib.

\section{Introduction}

Lung cancer is one of the most typical cancers worldwide, with more than 2 million new cases and over 1.7 million deaths each year [1], of which nonsmall cell lung cancer (NSCLC) accounts for 85\% of all lung cancers [2]. EGFR mutations are known to be carcinogenic driver mutations that occur in $10-44 \%$ of lung adenocarcinomas. In recent decades, apart from traditional treatments such as surgery, chemotherapy, and radiotherapy, epidermal growth factor receptor- (EGFR-) tyrosine kinase inhibitors (TKIs) have been widely used in the treatment of NSCLC patients [3]. In addition to enhancing progression-free survival of patients, EGFR-TKIs are less toxic than chemotherapy [4]. Previous studies have reported that gefitinib, the first-generation of EGFR-TKIs, is effective against tumors with EGFR activation mutations including EGFR L858R and EGFR del-19 $[5,6]$. Nevertheless, even if NSCLC patients harboring gene mutations initially respond to gefitinib, drug resistance inevitably develops [7].
Toschi et al. believed that NSCLC resistance to firstgeneration EGFR-TKIs was related to aberrant cell-mesenchymal epidermal transformation factor (c-Met) activity [8]. c-Met has been proven to be the only high proreceptor that binds to the hepatocyte growth factor (HGF). Abnormal c-Met activity in NSCLC can be elevated by MET gene mutation and amplification or upregulated HGF expression. After the binding of HGF to c-Met, autophosphorylation of c-Met activates a variety of intracellular signaling pathways, promoting tumor progression, invasion, and metastasis [9]. In lung cancer with aberrant c-Met activity leading to gefitinib resistance, sensitivity to gefitinib may be restored through suppression of c-Met signaling [7].

Currently, traditional Chinese medicine has attracted much attention because of its potent application in anticancer treatment. Cinobufotalin (huachansu) is extracted from the skin secretions of Bufo gargarizans, with benefits of detoxification, promoting blood circulation and removing blood stasis [10]. Several studies have shown that cinobufotalin can regulate immune function and promote 
apoptosis of tumor cells [11]. Cinobufotalin combined with chemotherapeutic agents has shown potent anticancer effects in a variety of cancers, such as liver cancer, pancreatic cancer, lung cancer, and hepatocellular carcinoma [12]. However, it has not been determined whether cinobufotalin in combination with gefitinib can be used for the treatment of lung cancer.

Herein, this study evaluated the efficacy of cinobufotalin combined with gefitinib on the growth of human lung adenocarcinoma A549 cells, providing a new therapeutic therapy for the treatment of NSCLC.

\section{Materials and Methods}

2.1. Cell Culture and Drug Treatment. Human nonsmall cell lung adenocarcinoma A549 cell strain and normal human lung BEAS-2B cell strain were provided by the Third Affiliated Hospital of Xinjiang Medical University Cancer Institute. Cinobufotalin and gefitinib were purchased from Solarbio and Sinopharm, respectively. A549 and BEAS2B cells were cultured in RPMI1640 medium containing $10 \%$ fetal bovine serum (Invitrogen), $100 \mathrm{U} / \mathrm{mL}$ penicillin, and $100 \mathrm{U} / \mathrm{mL}$ streptomycin at $37^{\circ} \mathrm{C}$ in a humidified atmosphere of $5 \% \mathrm{CO}_{2}(\mathrm{pH} 7.2-7.4)$. The cells at the logarithmic growth stage were divided into the control group (DMSO), gefitinib group $(1,5,10,20$, and $40 \mu \mathrm{mol} / \mathrm{L})$, cinobufotalin group $(0.005,0.01,0.05,0.1$, and $0.5 \mathrm{mg} / \mathrm{mL})$, and cinobufotalin plus gefitinib group.

2.2. MTT Assay. The cell density of both A549 and BEAS-2B was adjusted to $5 \times 10^{4} / \mathrm{mL}$ and inserted into a 96-well plate, followed by the addition of cell suspension $100 \mu \mathrm{L}$. At 24, 48, and $72 \mathrm{~h}$, MTT solution $10 \mu \mathrm{L}$ (Wuhan Biofavor Biotechnology Service Co., Ltd.) was added for further culture for $4 \mathrm{~h}$, and the medium was sucked out. The formazan crystals were dissolved in DMSO $150 \mu \mathrm{L}$. The blank pores were used as blank groups. The absorbance (OD) at $568 \mathrm{~nm}$ was measured by the microplate reader. The experiment was repeated three times.

2.3. Apoptosis and Cell Cycle Assays. A549 cells were inoculated in a 6 -well plate at a density of $2 \times 10^{5}$ cells per well, and cultured in an incubator of $5 \% \mathrm{CO}_{2}$ at $37^{\circ} \mathrm{C}$ for $48 \mathrm{~h}$. After incubation, cells were digested with $0.25 \%$ trypsin without EDTA and then rinsed with PBS twice and resuspended in the $500 \mu \mathrm{L}$ of binding buffer. Subsequently, cells were mixed with annexin-V-FITC $5 \mu \mathrm{L}$ and propidium iodide (PI) $5 \mu \mathrm{L}$ (Nanjing KeyGen Biotech Co., Ltd.) in the dark at room temperature for 5-15 min. Cell apoptosis rate and cell cycle were detected by flow cytometry (CytoFLEX, BECKMAN).

2.4. TUNEL Staining. TdT-mediated dUTP nick-end labeling (TUNEL) apoptosis detection kit was bought from Roche Applied Science Company. Cultured cells were immersed in $4 \%$ paraformaldehyde ( $\mathrm{pH} 7.4$ ) solution at room temperature, followed by $0.1 \%$ PBS solution for $2 \mathrm{~min}$.
TUNEL reaction mixture $50 \mu \mathrm{L}$ was added in each group, and the slides were incubated at $37^{\circ} \mathrm{C}$ for $60 \mathrm{~min}$ in the dark. Notably, the control group was only added with luciferinlabeled dUTP solution $50 \mu \mathrm{L}$. DAPI was added for incubation in dark for $5 \mathrm{~min}$. Subsequently, the slides were sealed with an antifluorescent quenching agent, and the images were analyzed under a fluorescence microscope.

2.5. ROS Detection. A549 cells were incubated with $5 \mu \mathrm{M}$ of dichlorodihydrofluorescein diacetate (DCFH-DA) fluorescent probe for $20 \mathrm{~min}$ in the dark. DCFH-DA can be hydrolyzed by esterases to a nonfluorescent molecule 2,7dichlorofluorescin and oxidized into fluorescent molecule 2,7-dichlorofluorescin in the presence of ROS. Then, the fluorescence emission was analyzed by a flow cytometer to detect the changes of ROS levels.

2.6. Western Blot. A549 cells were taken and washed with $3 \mathrm{~mL}$ of precooled PBS at $4^{\circ} \mathrm{C}$. A $400 \mu \mathrm{L}$ of lysis buffer containing PMSF (100 mmol/L) was added for lysis, and the cells were centrifuged at $12000 \mathrm{r} / \mathrm{min}$ at $4^{\circ} \mathrm{C}$ for $5 \mathrm{~min}$ to extract the total protein. Protein concentration was determined by the bovine serum albumin protein quantification method, with a loading of $40 \mu \mathrm{g}$ total proteins per well. Subsequently, an equal amount of proteins for each group were separated by SDS-PAGE and transferred onto PVDF membranes. The membranes were then blocked in 5\% skim milk in TBST and incubated overnight at $4^{\circ} \mathrm{C}$ with following primary antibodies: anti-Bax (rabbit polyclonal antibody, 00082363, Proteintech), anti-Bcl-2 (rabbit polyclonal antibody, 00083551, Proteintech), anti-cysteine-aspartic acid protease-3 (caspase-3, 15z0096, Affinity), anti-cyclin A (mouse polyclonal antibody, Proteintech), anti-cyclin E (rabbit polyclonal antibody, Proteintech), anti-P21 (rabbit polyclonal antibody, Proteintech), anti-cyclin-dependent kinase 2 (CDK2, rabbit polyclonal antibody, Proteintech), anti-MET (rabbit polyclonal antibody, Bioworld), anti-cMet (rabbit polyclonal antibody, Proteintech), and antiGAPDH (rabbit polyclonal antibody, AB-P-R001, Hangzhou Goodhere Co., Ltd.). The membranes were fully washed by TBST for 5-6 times and incubated with HRPlabeled sheep anti-rabbit secondary antibody (Wuhan Boster Biological Technology Co., Ltd.) at $37^{\circ} \mathrm{C}$ for $2 \mathrm{~h}$. Finally, bands were detected via ECL luminescence kit. Bandscan was used to analyze the protein gray value and calculate the relative expression of the target protein.

\subsection{Real-Time Fluorescence Quantitative PCR. Total RNA} from cells was extracted using TRIzol reagent. A microspectrophotometer was used to measure the OD260 value, OD280 value, and OD260/OD280 ratio of RNA, as well as the purity and concentration of RNA. RNA quality was estimated based on the OD260/OD280 ratio (ratio range: 1.8-2.0). The concentration of the sample RNA was calculated according to the following formula: total RNA concentration $(\mathrm{g} / \mathrm{L})=\mathrm{OD} 260 \times 40 \times 10^{-3}$. The total RNA was reverse transcribed to $\mathrm{cDNA}$, and the amplification and 
detection were performed by real-time quantitative PCR. The thermocycling conditions were set as follows: $50^{\circ} \mathrm{C}$ for $2 \mathrm{~min}$ and $95^{\circ} \mathrm{C}$ for $10 \mathrm{~min}$, followed by 40 cycles at $95^{\circ} \mathrm{C}$ for $30 \mathrm{~s}$ and $60^{\circ} \mathrm{C}$ for $30 \mathrm{~s}$. Final data were calculated with the ${ }_{2-}{ }^{\triangle \triangle} \mathrm{Ct}$ method. The primer sequence is given in Table 1.

\section{Statistical Analysis}

SPSS 22.0 software was used for statistical analysis. Measurement data were expressed as mean \pm standard deviation. The LSD $t$-test was used for comparison between every two groups, and analysis of variance (ANOVA) in repeated measurement was adopted for comparison among multiple groups. $P<0.05$ was considered statistically significant.

\section{Results}

4.1. Combination of Cinobufotalin and Gefitinib Decreases the Viability of A549 Cells. From the results of MTT assay, gefitinib alone $(1,5,10,20$, and $40 \mu \mathrm{mol} / \mathrm{L})$ or combined with cinobufotalin significantly decreased the viability of A549 cells in a dose- and time-dependent manner (Figure 1). The half-maximal inhibitory concentration (IC50) of gefitinib was $24.53 \mu \mathrm{mol} / \mathrm{L}$ at $24 \mathrm{~h}$ and $3.23 \mu \mathrm{mol} / \mathrm{L}$ at $72 \mathrm{~h}$ in the combination group, which was lower than gefitinib alone $(31.61 \mu \mathrm{mol} / \mathrm{L}$ at $24 \mathrm{~h}$ and $6.61 \mu \mathrm{mol} / \mathrm{L}$ at $72 \mathrm{~h})$. However, the IC50 value of gefitinib alone in BEAS-2B cells was higher than that in A549 cells (IC50 $=17.12 \mu \mathrm{M}$ vs. IC50 $=6.61 \mu \mathrm{M})$, indicating that the combined treatment of cinobufotalin and gefitinib was less toxic to normal cells (Figure 1(d)). Based on these results, gefitinib $40 \mu \mathrm{mol} / \mathrm{L}$ and cinobufotalin $0.5 \mathrm{mg} / \mathrm{mL}$ were chosen for subsequent experiments.

4.2. Combination of Cinobufotalin and Gefitinib Induces A549 Cell Apoptosis. We detected the A549 cell apoptosis under different treatments via flow cytometry and TUNEL assay. The apoptosis rate of A549 cells increased from $4.46 \% \pm 0.65 \%$ in control to $14.76 \% \pm 0.48 \%, 9.34 \% \pm 0.37 \%$, and $44.8 \% \pm 0.62 \%$ with treatment of gefitinib $40 \mu \mathrm{mol} / \mathrm{L}$, cinobufotalin $0.5 \mathrm{mg} / \mathrm{mL}$, and gefitinib plus cinobufotalin, respectively (Figures $2(\mathrm{a})$ and $2(\mathrm{~b})$ ). Subsequently, TUNEL assay was performed to further confirm the apoptotic effect of the combination therapy. Compared with the control group, cell apoptosis of each experimental group was remarkably elevated. In addition, the apoptosis rate of A549 cell in the combined treatment group was higher than that of the gefitinib group and cinobufotalin group (Figure 2(c)). In Western blot, the expression of Bax and caspase- 3 in the A549 cells treated with gefitinib plus cinobufotalin was enhanced, while Bcl-2 expression was significantly downregulated (all $P<0.0001$ ) (Figure $2(\mathrm{~d})$ ).

4.3. Combination of Cinobufotalin and Gefitinib Inhibits A549 Cell Cycle in the S Phase. Next, we performed cell cycle assay on A549 cells and found an increase of cell accumulation in the $S$ phase, but a reduction in the G2 phase after treatment with gefitinib and cinobufotalin either alone or in combination (Figures 3(a) and 3(b)). Besides, the cell number in the $S$ phase of the gefitinib + cinobufotalin group was higher than that of the gefitinib group $(P<0.05)$. As expected, the cell number in the G2 phase in gefitinib combined with the cinobufotalin group was significantly lower than that in gefitinib and cinobufotalin alone groups $(P<0.0001)$. Then, Western blot was performed to investigate the cell cyclerelated proteins. The combined therapy of gefitinib and cinobufotalin significantly downregulated the expression of cyclin A, cyclin E, and CDK2 $(P<0.0001)$ (Figure 3(c)). On the contrary, P21 expression in the A549 cells was upregulated after treatment with cinobufotalin plus gefitinib $(P<0.0001)$.

4.4. Cinobufotalin in Combination with Gefitinib Promotes ROS Production. To further confirm the involvement of ROS during cinobufotalin combined with gefitinib treatment, ROS generation was analyzed in A549 cells via DCFHDA staining, followed by flow cytometry. As shown in Figure 4(a), compared with the control group, the ROS production in combination with the combined gefitinib group was obviously higher than that in cinobufotalin alone or gefitinib alone $(P<0.0001)$.

4.5. Cinobufotalin in Combination with Gefitinib Suppressed $H G F$ and $c-M e t$ Expression. The underlying mechanism that resulted in the superior effect of gefitinib combined with cinobufotalin was evaluated. Treatment with cinobufotalin $0.5 \mathrm{mg} / \mathrm{mL}$ or gefitinib $40 \mu \mathrm{mol} / \mathrm{L}$ alone or in combination (cinobufotalin + gefitinib group) significantly inhibited HGF/GAPDH and c-Met/GAPDH ratios to 0.4765 and 0.62857 , respectively (Figure 4(b)). Compared with the gefitinib group, the protein levels of HGF and c-Met were further declined in the combination group $(P<0.01$ and $P<0.0001$, respectively). QRT-PCR analysis was performed to detect gene amplification of c-Met in A549 cells and found a significant reduction of c-Met gene amplification in the combination group (Figure 4(c)).

\section{Discussion}

The most notable finding of this study was that cinobufotalin combined with gefitinib enhanced A549 cell apoptosis and inhibited cell viability and cell cycle by downregulating HGF and c-Met protein expression. This finding fills the gap in the impact of combination of cinobufotalin and gefitinib on lung cancer. Targeted therapy has been a study focus in the field of cancer in the past decade. In the molecular biology research of NSCLC, EGFR mutation is a molecular target of general concern in medical research, and EGFR-TKIs such as gefitinib and erlotinib have been widely applied in clinical practice [13-15]. Although gefitinib has a certain beneficial function on human malignancy, it can also result in greater toxicity by killing normal cells. Moreover, the median time for many patients to develop resistance to gefitinib is about 10 months [16]. Therefore, it is of great urgency to find out a novel strategy to delay or overcome the acquired resistance to gefitinib. 
Table 1: Primer sequences.

\begin{tabular}{lcc}
\hline Primer & Primer sequence $\left(5^{\prime} \longrightarrow 3^{\prime}\right)$ & Length $(\mathrm{bp})$ \\
\hline \multirow{2}{*}{ Homo GAPDH } & Forward: 5 “- TCAAGAAGGTGGTGAAGCAGG -3” & 115 \\
\hline \multirow{2}{*}{ Homo c-Met } & Reverse: 5 “- TCAAAGGTGGAGGAGTGGGT -3” & 240 \\
\hline
\end{tabular}

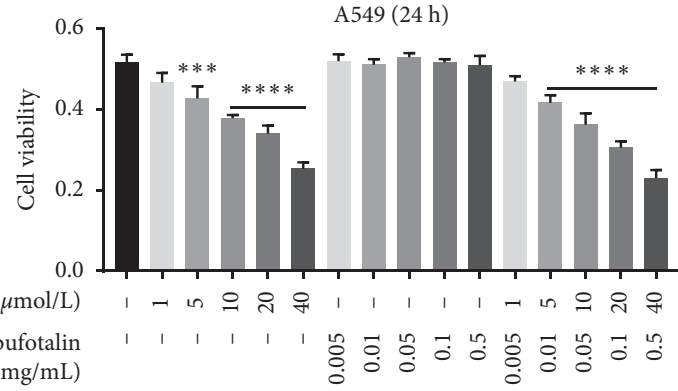

(a)

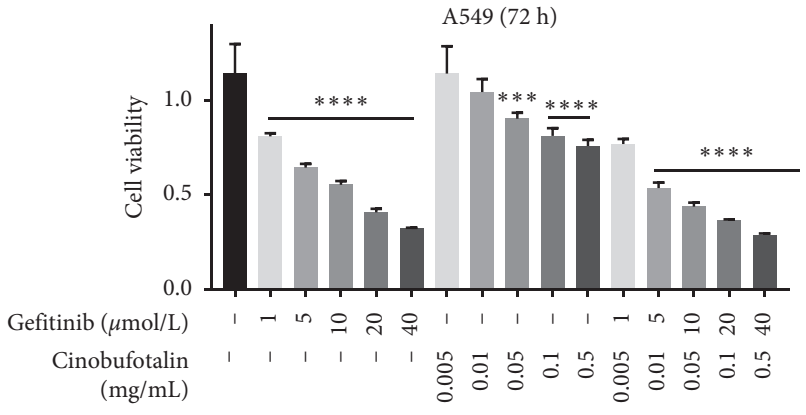

(c)

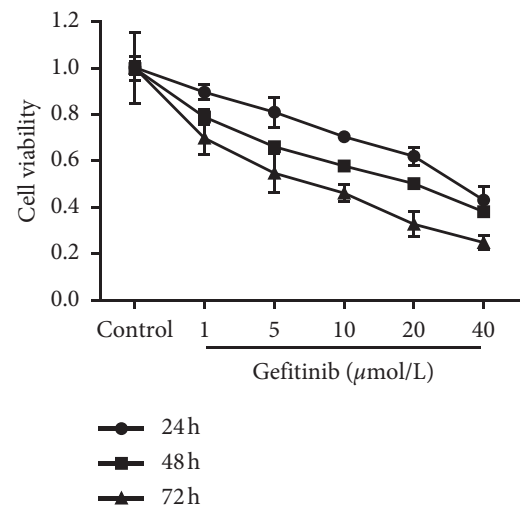

(e)

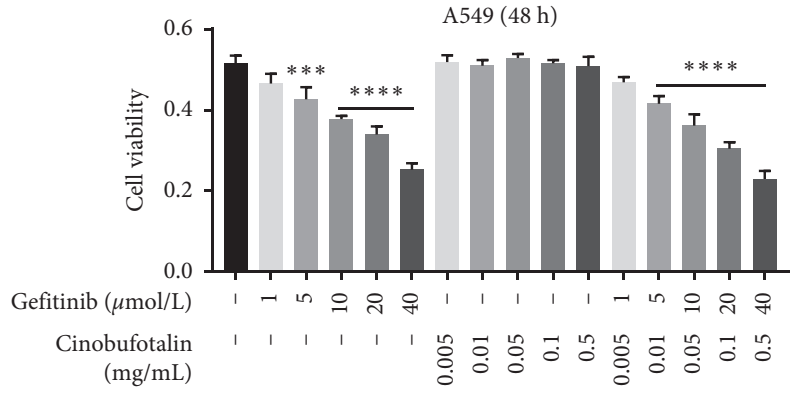

(b)

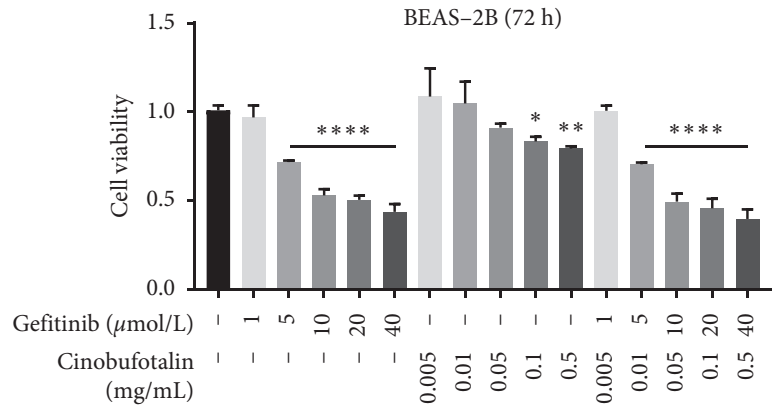

(d)

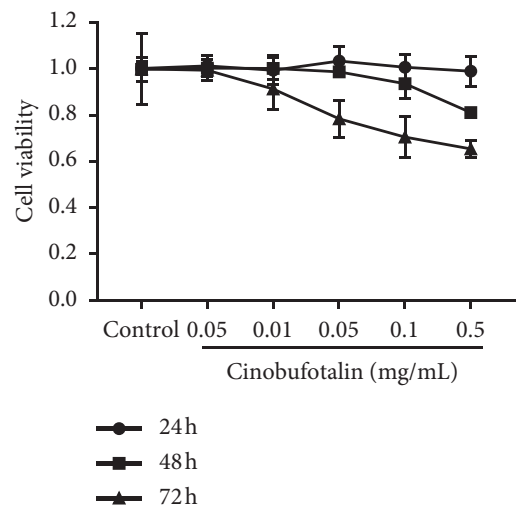

(f)

FIGURE 1: Cinobufotalin and gefitinib decreased A549 cell viability. MTT assay was performed to evaluate the inhibitory effect of gefitinib and cinobufotalin on A549 cells viability at (a) $24 \mathrm{~h}$, (b) $48 \mathrm{~h}$, and (c) $72 \mathrm{~h}$ either alone or in combination. (d) MTT assay was applied to assess the inhibitory function of gefitinib and cinobufotalin on BEAS-2B cell viability at $72 \mathrm{~h}$ either alone or in combination. The inhibitory effect of (e) gefitinib alone $(1,5,10,20$, and $40 \mu \mathrm{mol} / \mathrm{L})$ and (f) cinobufotalin alone $(0.005,0.01,0.05,0.1$, and $0.5 \mathrm{mg} / \mathrm{mL})$ on A549 cell viability were also detected. ${ }^{*} P<0.05,{ }^{* *} P<0.01,{ }^{* * *} P<0.001$, and ${ }^{* * * *} P<0.0001$ vs. the control group (untreated cells). ${ }^{*} P<0.05$ vs. the gefitinib group at the corresponding drug concentration. 

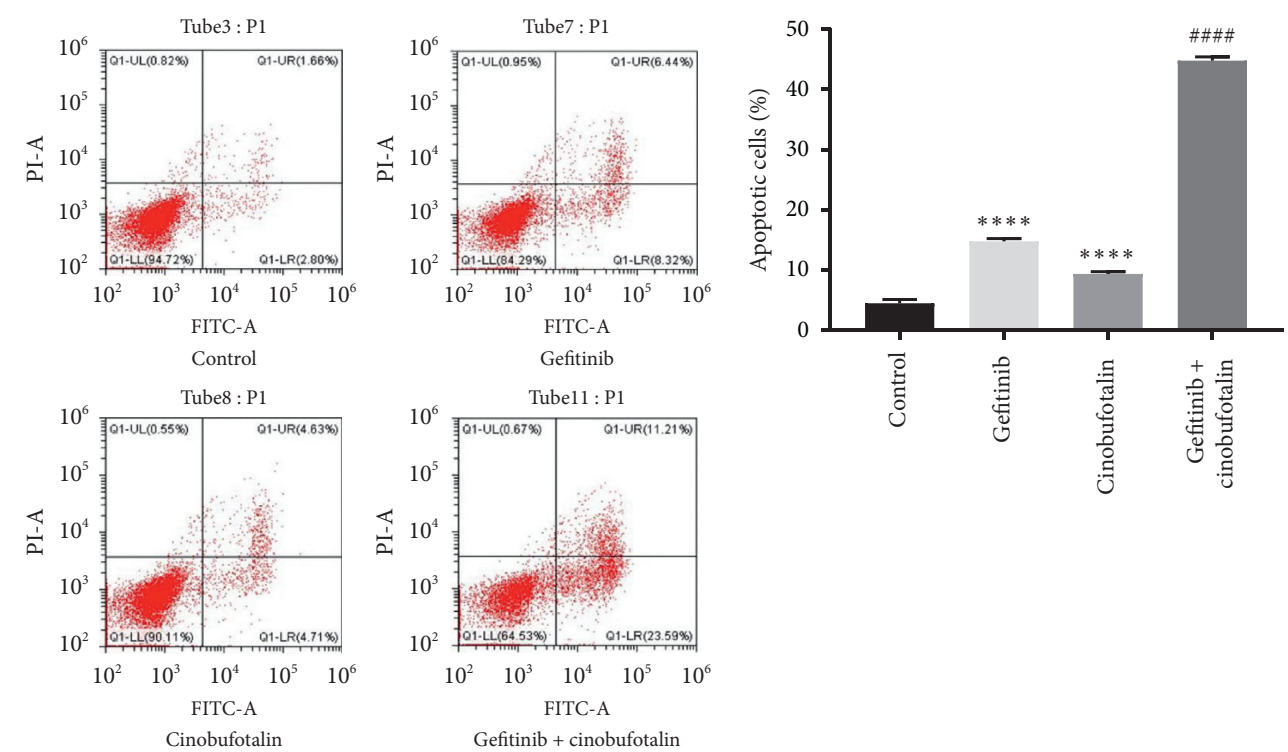

(a)

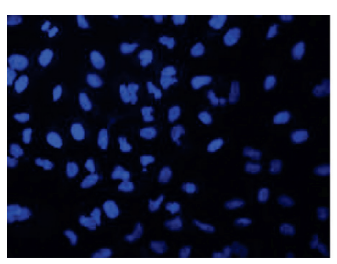

Control

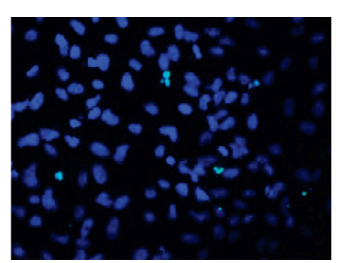

Gefitinib

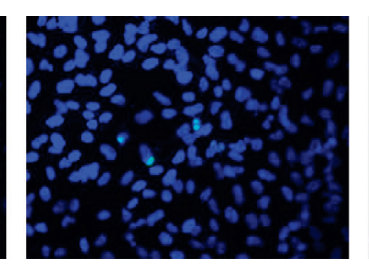

Cinobufotalin

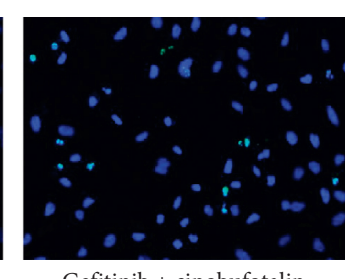

Gefitinib + cinobufotalin

(c)

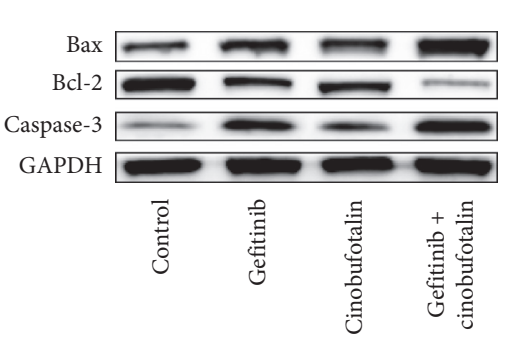

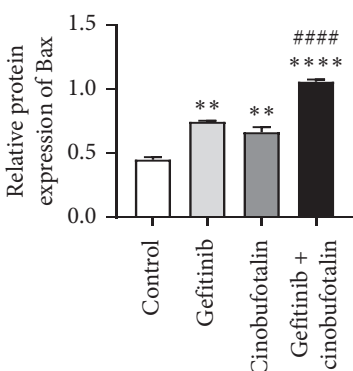

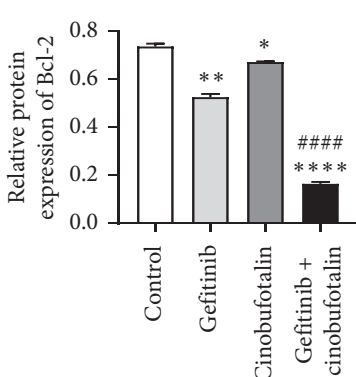

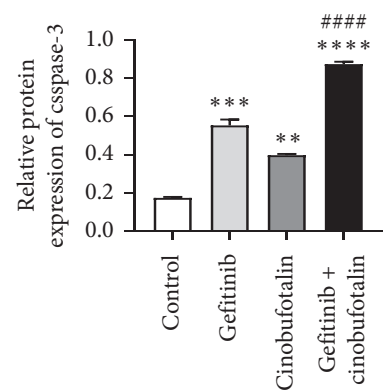

(d)

Figure 2: Combination of cinobufotalin and gefitinib induces A549 cells apoptosis. (a) A549 cells were treated with gefitinib $40 \mu \mathrm{mol} / \mathrm{L}$ and cinobufotalin $0.5 \mathrm{mg} / \mathrm{mL}$ for $48 \mathrm{~h}$ either alone or in combination. Apoptotic rate of A549 cells was evaluated by annexin-V/PI staining and flow cytometry. (b) The percentage of apoptotic cells in the treatment groups was quantified. (c) TUNEL staining of A549 cells. A549 cells were treated with gefitinib $40 \mu \mathrm{mol} / \mathrm{L}$ and cinobufotalin $0.5 \mathrm{mg} / \mathrm{mL}$ either alone or in combination and subsequently stained with TUNEL (magnification $\times 200$ ). Green, apoptotic cells; blue, nucleus. (d) Western blot analysis of cell apoptosis-related proteins in A549 cells treated with gefitinib, cinobufotalin, or cinobufotalin + gefitinib. ${ }^{*} P<0.05,{ }^{* *} P<0.01,{ }^{* * *} P<0.001$, and ${ }^{* * * *} P<0.0001$ vs. the control

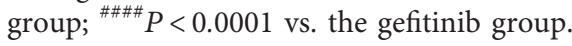

Cinobufotalin is an animal-derived drug for the treatment of human malignancies. Qian et al. have implied that cinobufotalin regulates the expression of apoptosis-related protein Mcl-1 and invasion-related proteins (E-cadherin, MMP9, and Snail) by inhibiting c-Met expression in gallbladder cancer [17]. In addition, cinobufotalin can suppress gastric cancer cell proliferation and promotes cell apoptosis [12]. Although several studies have suggested that cinobufotalin has the potential to be a novel anticancer agent, little is known about the role of cinobufotalin combined with gefitinib in the treatment of lung cancer. In the current study, A549 cells were treated with cinobufotalin alone and found that cinobufotalin dramatically impeded the cell viability in a dose- and time-dependent manner. Several studies have confirmed prominent therapeutic efficacy of cinobufotalin combined with chemotherapy for NSCLC, which is more effective than chemotherapy alone [18]. Consistently, A549 cell viability in the combination group 

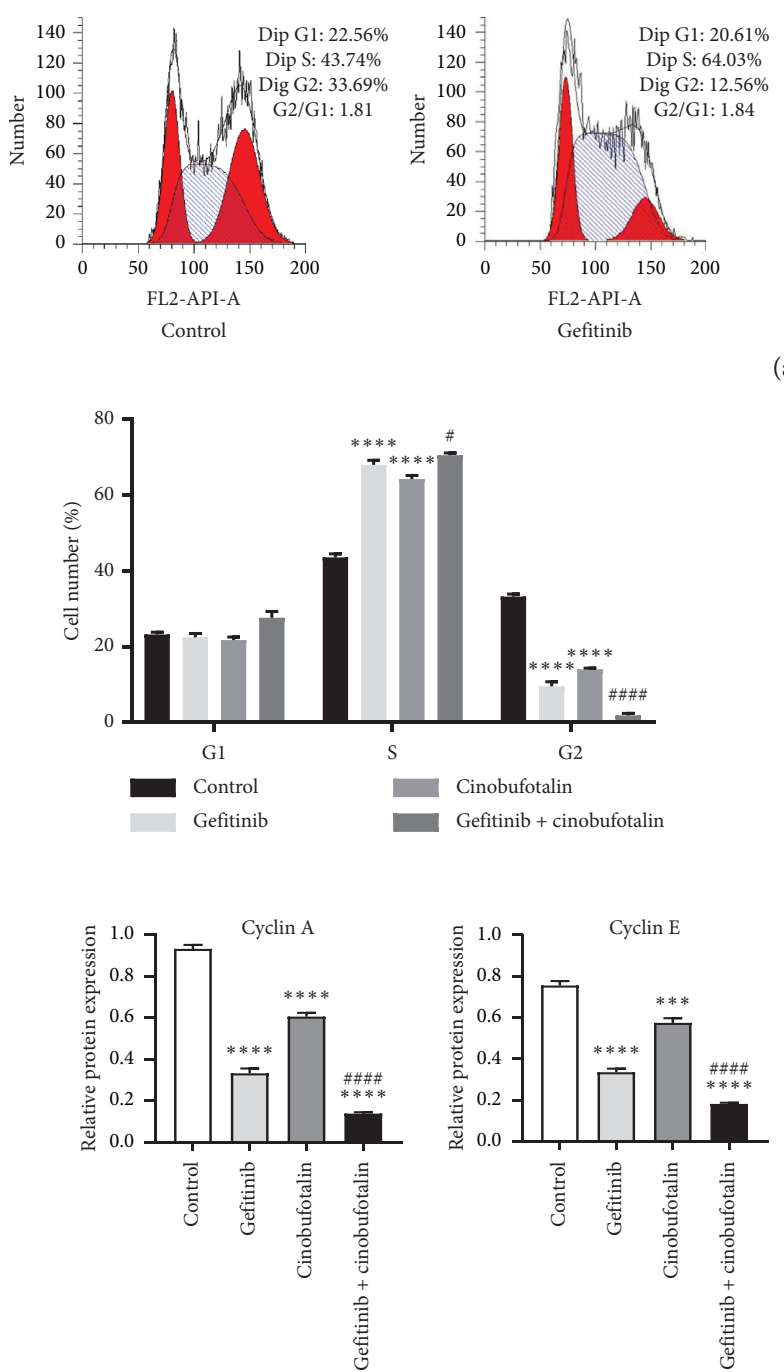

(b)
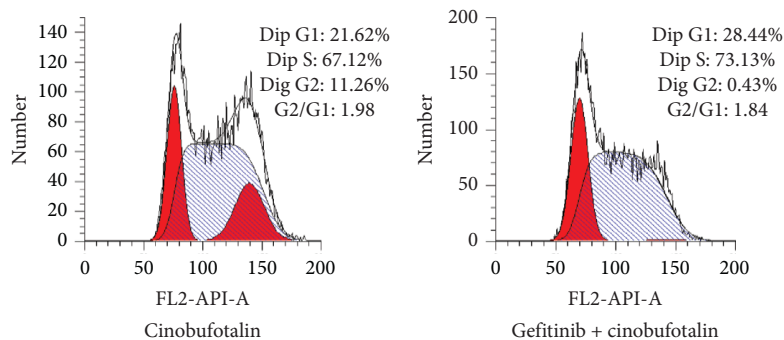

(a)
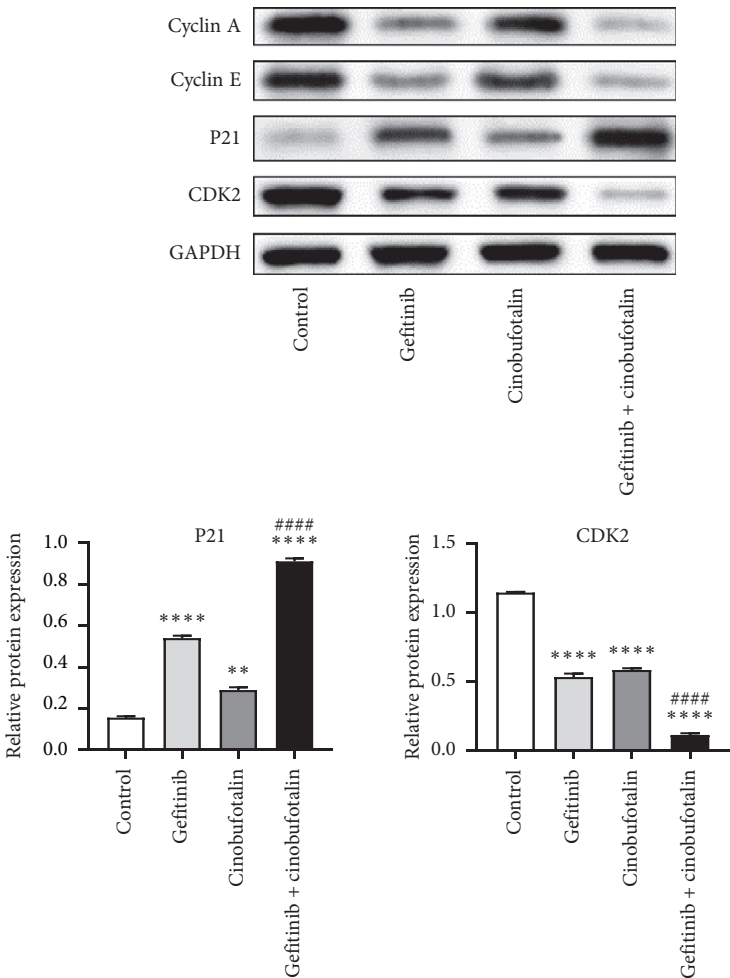

(c)

FIgURE 3: Combination of cinobufotalin and gefitinib inhibits A549 cell cycle in the S phase. (a) Flow cytometry data of cell cycle analysis. (b) Representative quantification of cell cycle analysis data indicating percentage of cells in given phases. (c) Western blot analysis of cell cycle control proteins in A549 cells in the presence of gefitinib, cinobufotalin, or cinobufotalin + gefitinib. ${ }^{* *} P<0.01,{ }^{* * *} P<0.001$, and ${ }^{* * * * *} P<0.0001$ vs. the control group; ${ }^{\#} P<0.05$ and ${ }^{\# \# \# \#} P<0.0001$ vs. the gefitinib group.

was significantly lower than that of the gefitinib group, indicating that cinobufotalin played a certain synergistic effect in combination with gefitinib.

The results of annexin-V/PI staining and flow cytometry revealed that the apoptosis of A549 cells in the gefitinib + combination group was significantly higher than that in the single treatment group, which was further confirmed by TUNEL assay. Activated proapoptotic proteins such as Bax result in the release of the apoptogenic cytochrome-c from the mitochondrial membrane, causing caspase cascade activation [19]. In this study, Bax and caspase-3 expression was elevated in A549 cells treated with gefitinib plus cinobufotalin, while the expression of antiapoptotic protein Bcl-2 was downregulated. Notably, compared with the normal group, the cell cycle of the gefitinib group and the cinobufotalin group was blocked in the $\mathrm{S}$ phase, and the blocking effect of the combined group was significantly enhanced, thus delaying cell mitosis. Besides, we also noticed downregulated expression of cyclin A, cyclin E, and CDK2 and upregulated expression of cyclin-dependent kinase inhibitor P21 in the gefitinib + cinobufotalin group. A recent report described that cinobufotalin induced a ROS-dependent autophagy cell death in lymphoma cells [19]. We speculated that ROS might be involved in the mechanism of gefitinib combined with cinobufotalin to lung cancer cell apoptosis. To verify the idea, DCFH-DA staining was performed to evaluate the ROS generation, and results showed that the induction of cell apoptosis by the combined therapy was linked to the generation of ROS. These data demonstrated that cinobufotalin potentiated the sensitivity of A549 cells to anticancer drug gefitinib, thereby promoting cell apoptosis.

In recent years, studies have found that some patients develop primary resistance to gefitinib or acquired resistance 

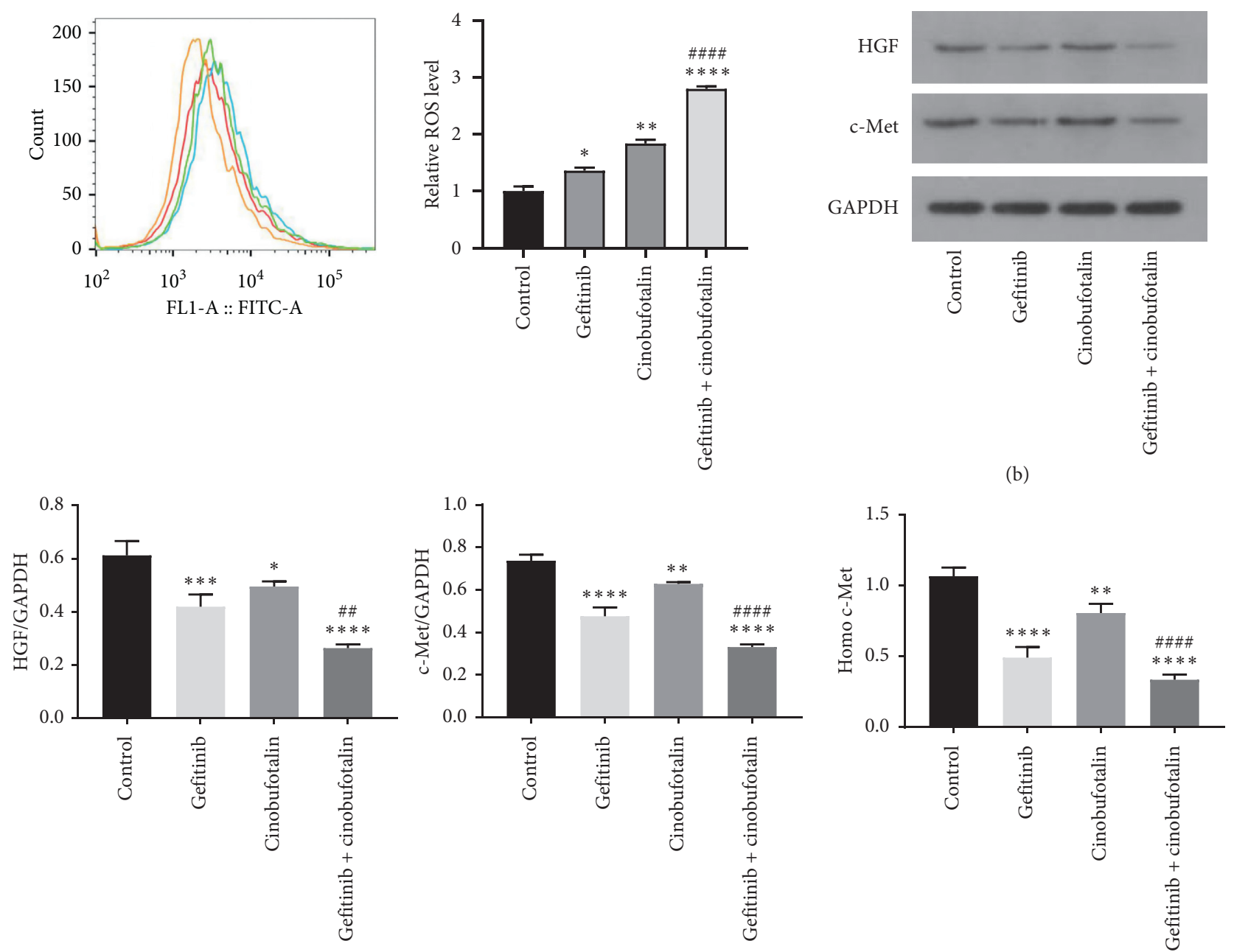

(a)

(c)

FIGURE 4: Combination of cinobufotalin and gefitinib induced ROS accumulation and suppressed the expression of HGF and c-Met. (a) ROS production was increased after combined treatment with cinobufotalin and gefitinib. (b) A549 cells were analyzed for HGF and cMet protein expression by Western blot. (c) c-Met gene expression was determined by real-time quantitative PCR. ${ }^{*} P<0.05,{ }^{*} * P<0.01$, ${ }^{* * *} P<0.001$, and ${ }^{* * * *} P<0.0001$ vs. the control group; ${ }^{\# \#} P<0.01$ and ${ }^{\# \# \# \#} P<0.0001$ vs. the gefitinib group. The experiment was repeated three times.

to gefitinib within $8-10$ months, affecting progression-free and overall survival $[20,21]$. A study conducted by Lu et al. pointed out that gefitinib resistance might be related with the activation of downstream EGFR signaling pathways such as $\mathrm{MAPK} / \mathrm{c}-$ Fos and AKT/Bcl-2; besides, c-Met overexpression could also greatly limit the EGFR-targeted therapy [22]. The c-Met protooncogene can be amplified by bypassing the inhibited EGFR-phosphorylated kinase pathway through the ERBB3-PI3K-Akt and MAPK-ERk1/2T pathways. The amplified c-Met avoids killing EGFR-TKIs and promotes the proliferation of cancer cells by promoting downstream signal transduction through bypass activation and ultimately leads to drug resistance of patients to EGFR-TKIs [23]. Additionally, lung cancer cells can secrete a variety of cytokines to promote the continuous secretion of HGF by peripheral fibroblasts, thus forming a positive feedback loop, leading to the infinite growth of cancer cells [24]. Cancer cells with high c-Met expression are more sensitive and invasive to HGF. c-Met amplification is independent of the EGFR pathway, and $10 \%$ of EGFR-TKIS resistance is completely caused by c-Met gene amplification [25]. Our results showed that the combination of gefitinib and cinobufotalin remarkably downregulated the protein expression of c-Met and HGF, which further suggested that cinobufotalin could prevent the vicious cycle of the HGF/c-Met pathway to a certain extent.

In tumor cell lines with c-Met gene amplification, the growth of tumor cells depends on the c-Met gene signaling pathway. However, there is a certain relationship between the acquired resistance of EGFR-TKIS and c-MET gene amplification in about $20 \%$ of tumor cell lines without c-Met gene amplification [26]. In this study, the amplification of c-Met gene was noticeably impeded in gefitinib and cinobufotalin groups, which was further blocked by the combined treatment of gefitinib and cinobufotalin. Overall, these findings suggested that cinobufotalin not 
only elevated the sensitivity of lung cancer to gefitinib but also inhibited gene amplification of c-Met to a certain extent. The main limitation of this research is that the pathway mechanism of gefitinib combined with cinobufotalin against lung cancer remains unclear, and we will further investigate its potential downstream pathway mechanism in the future.

\section{Conclusion}

Cinobufotalin combined with gefitinib suppresses A549 cell viability and promotes cell apoptosis by downregulating HGF protein expression and blocking c-Met gene amplification, indicating that cinobufotalin may delay the occurrence of gefitinib resistance in lung cancer cells. In summary, this study provides a novel idea for the future study of the treatment of gefitinib-resistant lung cancer with cinobufotalin.

\section{Data Availability}

The data used and/or analyzed during the current study are available from the corresponding author upon request.

\section{Conflicts of Interest}

The authors declare that there are no conflicts of interest.

\section{Authors' Contributions}

Youxi Han and Ronghui Ma contributed equally to this work.

\section{Acknowledgments}

This study was supported by the National Natural Science Foundation of China (81760014), Xinjiang Autonomous Region Science and Technology Support Plan Project (2019E0281), Tianshan Youth Program (2018Q048), and Natural Science Foundation of Xinjiang Uygur Autonomous Region (2017D01C411).

\section{References}

[1] J. Ferlay, M. Colombet, I. Soerjomataram et al., "Estimating the global cancer incidence and mortality in 2018: GLOBOCAN sources and methods," International Journal of Cancer, vol. 144, no. 8, pp. 1941-1953, 2019.

[2] T. Lu, X. Yang, Y. Huang et al., "Trends in the incidence, treatment, and survival of patients with lung cancer in the last four decades," Cancer Management and Research, vol. 11, pp. 943-953, 2019.

[3] M. Takeda and K. Nakagawa, "First- and second-generation EGFR-TKIs are all replaced to osimertinib in chemo-naive EGFR mutation-positive non-small cell lung cancer?" International Journal of Molecular Sciences, vol. 20, no. 1, p. 146, 2019.

[4] J. C. Yang, V. Hirsh, M. Schuler et al., "Symptom control and quality of life in LUX-Lung 3: a phase III study of afatinib or cisplatin/pemetrexed in patients with advanced lung adenocarcinoma with EGFR mutations," Journal of Clinical Oncology, vol. 31, no. 27, pp. 3342-3350, 2013.
[5] Y. L. Wu, Y. Cheng, X. Zhou et al., "Dacomitinib versus gefitinib as first-line treatment for patients with EGFR-mutation-positive non-small-cell lung cancer (ARCHER 1050): a randomised, open-label, phase 3 trial," The Lancet Oncology, vol. 18, no. 11, pp. 1454-1466, 2017.

[6] M. Friese-Hamim, F. Bladt, G. Locatelli, U. Stammberger, and A. Blaukat, "The selective c-Met inhibitor tepotinib can overcome epidermal growth factor receptor inhibitor resistance mediated by aberrant c-Met activation in NSCLC models," American Journal of Cancer Research, vol. 7, no. 4, pp. 962-972, 2017.

[7] J. A. Engelman, K. Zejnullahu, T. Mitsudomi et al., "MET amplification leads to gefitinib resistance in lung cancer by activating ERBB3 signaling," Science, vol. 316, no. 5827, pp. 1039-1043, 2007.

[8] L. Toschi and F. Cappuzzo, "Clinical implications of MET gene copy number in lung cancer," Future Oncology, vol. 6, no. 2, pp. 239-247, 2010.

[9] N. Van Der Steen, K. Zwaenepoel, G. Mazzaschi et al., “The role of c-met as a biomarker and player in innate and acquired resistance in non-small-cell lung cancer: two new mutations warrant further studies," Molecules, vol. 2424 pages, 2019.

[10] S. Kai, J. H. Lu, P. P. Hui, and H. Zhao, "Pre-clinical evaluation of cinobufotalin as a potential anti-lung cancer agent," Biochemical and Biophysical Research Communications, vol. 452, no. 3, pp. 768-774, 2014.

[11] F. Zhang, Y. Yin, and T. Xu, "Cinobufotalin injection combined with chemotherapy for the treatment of advanced NSCLC in China: a PRISMA-compliant meta-analysis of 29 randomized controlled trials," Medicine (Baltimore), vol. 98, no. 35, Article ID e16969, 2019.

[12] G. Wang, G. Liu, Y. Ye, Y. Fu, and X. Zhang, "Bufothionine exerts anti-cancer activities in gastric cancer through Pim3," Life Sciences, vol. 232, p. 116615, 2019.

[13] F. Wang, X. Y. Diao, X. Zhang et al., "Identification of genetic alterations associated with primary resistance to EGFR-TKIs in advanced non-small-cell lung cancer patients with EGFR sensitive mutations," Cancer Communication (London), vol. 39, no. 1, p. 7, 2019.

[14] P. Hulo, D. Coupez, M. G. Denis, and J. Bennouna, "EGFR mutation-positive NSCLC: factors to consider when deciding first-line therapy," Expert Review of Anticancer Therapy, vol. 20, no. 5, pp. 365-372, 2020.

[15] R. Li, X. Zhou, H. Yao, and L. Li, "Four generations of EGFR TKIs associated with different pathogenic mutations in nonsmall cell lung carcinoma," Journal of Drug Targeting, vol. 28, no. 9, pp. 861-872, 2020.

[16] D. Jackman, W. Pao, G. J. Riely et al., "Clinical definition of acquired resistance to epidermal growth factor receptor tyrosine kinase inhibitors in non-small-cell lung cancer," Journal of Clinical Oncology, vol. 28, no. 2, pp. 357-360, 2010.

[17] L. Qian, H. Su, G. Wang, B. Li, G. Shen, and Q. Gao, "Antitumor activity of bufalin by inhibiting c-MET mediated MEK/ ERK and PI3K/AKT signaling pathways in gallbladder cancer," Journal of Cancer, vol. 11, no. 11, pp. 3114-3123, 2020.

[18] B. Chen, "Clinical efficacy and anti-tumor mechanism of cinobufacini combined with GP regimen for advanced nonsmall cell lung cancer," Practical Journal of Cancer, vol. 31, pp. 224-227, 2016.

[19] H. Emam, Q. L. Zhao, Y. Furusawa et al., "Apoptotic cell death by the novel natural compound, cinobufotalin," ChemicoBiological Interactions, vol. 199, no. 3, pp. 154-160, 2012.

[20] H. N. Oh, M. H. Lee, E. Kim et al., "Licochalcone D induces ROS-dependent apoptosis in gefitinib-sensitive or resistant 
lung cancer cells by targeting EGFR and MET," Biomolecules, vol. 10 , no. 2 , p. $297,2020$.

[21] J. Li, X. H. Wang, J. Hu, M. Shi, L. Zhang, and H. Chen, "Combined treatment with $\mathrm{N}$-acetylcysteine and gefitinib overcomes drug resistance to gefitinib in NSCLC cell line," Cancer Medicine, vol. 9, no. 4, pp. 1495-1502, 2020.

[22] M. Lu, B. Liu, H. Xiong, F. Wu, C. Hu, and P. Liu, "Trans$3,5,4^{\prime}$-trimethoxystilbene reduced gefitinib resistance in NSCLCs via suppressing MAPK/Akt/Bcl-2 pathway by upregulation of miR-345 and miR-498," Journal of Cellular and Molecular Medicine, vol. 23, no. 4, pp. 2431-2441, 2019.

[23] O. Miranda, M. Farooqui, and J. M. Siegfried, "Status of agents targeting the HGF/c-Met axis in lung cancer," Cancers (Basel), vol. 10 , no. 9 , p. $280,2018$.

[24] T. Cascone, L. Xu, H. Y. Lin et al., "The HGF/c-MET pathway is a driver and biomarker of VEGFR-inhibitor resistance and vascular remodeling in non-small cell lung cancer," Clinical Cancer Research, vol. 23, no. 18, pp. 5489-5501, 2017.

[25] N. A. Joseph, S. H. Chiou, Z. Lung et al., "The role of HGFMET pathway and CCDC66 cirRNA expression in EGFR resistance and epithelial-to-mesenchymal transition of lung adenocarcinoma cells," Journal of Hematology \& Oncology, vol. 11, no. 1, p. 74, 2018.

[26] Y. H. Tseng, Y. C. Tseng, Y. H. Lin et al., "Epidermal growth factor receptor (EGFR)-Tyrosine kinase inhibitor treatment and salvage chemotherapy in EGFR-mutated elderly pulmonary adenocarcinoma patients," The Oncologist, vol. 20, no. 7, pp. 758-766, 2015. 